\title{
Virtual Circuit Management at IP-ATM Network Interface with Control Operating Policy
}

\author{
Mahbub Hassan ${ }^{a}$ and Mohammed Atiquzzaman ${ }^{b}$ \\ ${ }^{a}$ School of Computer Science \& Software Engineeringy \\ Monash University, Caulfield Campus \\ Caulfield, Melbourne, VIC 3145, Australia. \\ Tel: +61(3)99032122 Fax: +61(3)99031077 \\ Email: mahbub@insect.sd.monash.edu.au \\ ${ }^{b}$ Department of Electrical \& Computer Engineering \\ The University of Dayton, Dayton, Ohio 45469-0226, USA. \\ Tel: +1-937-229 3183, Fax: +1-937-229 4529 \\ Email: atiq@engr.udayton.edu
}

\begin{abstract}
ATM is a connection oriented network while IP is based on a connectionless protocol. Connecting the two networks requires protocol conversion. When IP packets arrive at the interconnection between the two networks, signalling is used to open a switched virtual circuit on the ATM side. In this paper, we look at the management of the ATM connection with a view to reducing the cost of the connection. We develop and compare the cost functions of three connections management policies: the Delayed Vacation Policy, the Control Operating Policy and the Permanent Virtual Circuit. Furthermore, we have developed the coditions under which the Control Operating Policy (COP) will be more cost effective than the other two policies. We conclude that there always exists some condition under which the COP will be better than the other two polcies.
\end{abstract}

Keywords: IP, ATM network, Queuing Theory, Control Operating Policy

\section{INTRODUCTION}

In the last few years, an enormours effort has been directed towards resolving various issues related to IP-ATM interworking. IP is based on a connectionless protocol whereas ATM is connection oriented. This requires that an ATM connection be opened at the IP-ATM gateway when an IP packet has to be transmitted over an ATM network (see Figure 1). One of the fundamental interworking issues is to find an efficient and cost effective solution for managing the opening and closing of ATM virtual circuits (VC) at the IP-ATM interface.

One simple way to address this issue is to open a permanent or semi-permanent virtual circuit (PVC) between every source and destination. This eliminates the need for opening a VC when an IP datagram needs to be transmitted over the ATM network. Although this solution has been used by many during the initial trial of ATM networks, it does not scale well for large networks and it wastes ATM bandwidth during idle periods when there is no IP datagram to be transmitted. The other solution is to use switched VC (SVC); open a VC when needed and close it when there is no data to transmit (see Figure 1). However, since there is some cost and delay incurred each time a VC is opened, it was suggested ${ }^{1}$ to leave a VC open (after the transmission of a datagram) for a while and set a timer. The VC is closed only if the timer expires. If an IP datagram arrives before the timer expires, the timer is cleared and a new timer is set when another idle period starts.

The main optimisation problem with the timer-based approach mentioned above is to select a suitable timeout interval for the timer. In one hand, the cost due to VC openings (VC opening cost) may become too much if the timeout interval is too small and in the other hand, the cost due to waste of VC bandwidth (VC running cost) may become significant if the timer is too large.

Keshav et al. ${ }^{2}$ studied the economic behaviour of the timer-based approach with the objective of minimising the $\mathrm{VC}$ operating cost by selecting the optimum timeout interval. However, Keshav et al. ignored the issue of queueing of IP datagrams at the IP-ATM interface. The queueing occures when IP datagrams arrive at the IP-ATM interface 


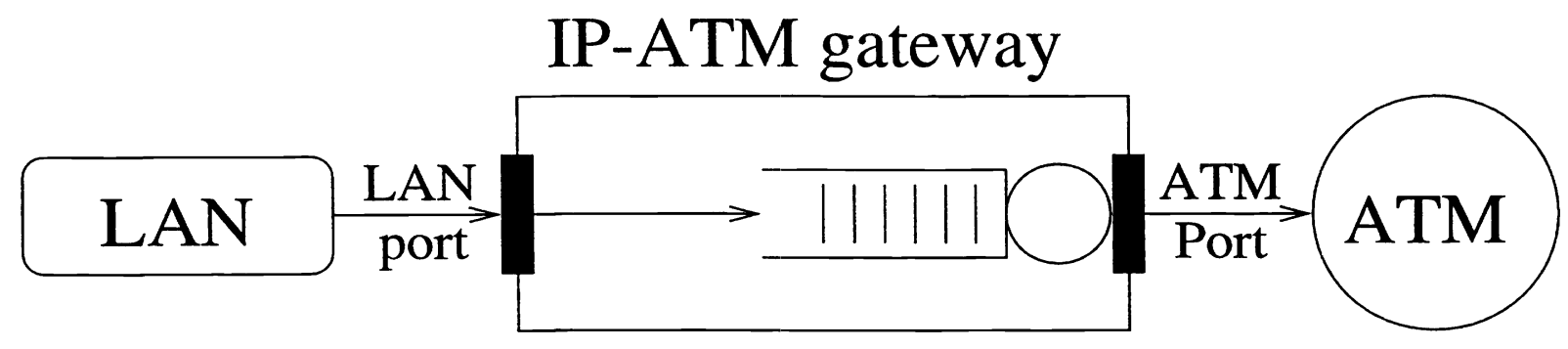

Figure 1. An IP LAN connected to an ATM network using a gateway.

during a VC opening or during the transmission of an IP datagram. Because queueing of datagrams use buffer space at the IP-ATM interface and increases the delay of datagrams, it introduces an additional cost component to the total VC operating cost. We refer to this cost as buffering cost.

Hassan and Atiquzzaman ${ }^{3}$ provided a queueing model, referred to as delayed vacation model, to study the queueing behaviour at the IP-ATM interface for the timer-based approach to VC management. The model in ${ }^{3}$ allows one to obtain the $\mathrm{VC}$ opening rate and the average queue length of the queueing system. Although accurate analysis was obtained in $^{3}$ for an $M / D / 1$ queue for the average queue length, only an approximate analysis was presented for an $M / M / 1$ queue. Later, Kitazume and Takahashi ${ }^{4,5}$ provided an exact analysis for the average queue length for an $\mathrm{M} / \mathrm{M} / 1$ queue with delayed vacation. We will refer to queueing systems controlled by the timer-based policy as delayed vacation policy (DVP).

The economic behaviour of an $M / G / 1$ queue under DVP was analysed in ${ }^{6}$ using the performance variables (VC opening rate and average queue length) obtained in, ${ }^{3,4}$ and was shown that for an $M / G / 1$ queue, the operating cost is minimised when the timeout interval is either zero or infinity depending on the VC opening cost, VC running cost and buffering (in the queue at the IP-ATM interface) cost.

The objective of this paper is to investigate alternative cost effective operating policies suitable for VC management at IP-ATM interface. One policy that consists of turning on and off the server is called control operating policy (COP) $\cdot^{7,8}$ Under COP, the server is turned off as soon as all the present datagrams are served, and turned on when the queue size (due to queued datagrams) reaches a threshold $K$ ( $K$ is the control parameter). The optimisation problem for COP is to find the value of $K$ (a positive integer) which minimises the total operating cost.

Heyman $^{7}$ studied the economic behaviour of $M / G / 1$ queueing systems under the COP and showed that COP is the optimal operating policy. However Heyman only considered zero 'startup time' which is equivalent to zero VC opening delay. Since the VC opening delay is expected to be significant for ATM networks, ${ }^{9,10}$ Heyman's results can not be applied to our case directly.

In this paper, we analyse the economic behaviour of COP for non-zero VC opening delay, and compare the effectiveness of the COP with DVP for an $M / M / 1$ queue. It is shown that, for certain cases, there exists an optimum $K$ greater than 1 which minimises the VC operating cost under COP. It is further shown that, for certain cases, COP achives lower operating cost than DVP.

The rest of the paper is organised as follows. All notations used throughout the paper are presented in Section 2. Economic analysis of DVP is summarised in Section 3. Cost functions for PVC and COP are discussed in Sections 4 and 5 respectively. Comparison between the three schemes is presented in Section 6 followed by concluding remarks in Section 7

\section{NO'TATIONS}

We define the following variables which are used to develop our model in Sections 3 and 5.

$\lambda$ : mean arrival rate of packets, i.e. the mean number of IP datagrams arriving at IP-ATM interface per unit time.

$\mu$ : mean service rate of packets, i.e. the mean number of IP datagrams that may be transmitted over the VC per unit time. 
$\rho$ : load on the IP-ATM interface $\left(\rho=\frac{\lambda}{\mu}\right)$

$S$ : VC opening delay, defined as the time required to open a VC (using signalling) when a packet arrives at the IP-ATM interface.

$T$ : timeout interval for DVP, defined as the time after which an idle VC will be closed down (using signalling).

$\hat{T}$ : the optimum value of $T$ which minimises the cost function of DVP.

$K$ : queue threshold in the COP, defined as the number of packets required in the queue to initiate the setting up of a new VC.

$\hat{K}$ : the optimum value of $K$ which minimises the cost function of the COP.

$L$ : average queue length, defined as the number of IP datagrams in the queue at the IP-ATM inteface plus the one being transmitted over the VC.

$\gamma$ : VC opening rate, defined as the number of times VCs need to be opened per unit time.

$\pi$ : fraction of time $\mathrm{VC}$ is running idle i.e., $\mathrm{VC}$ is open but not transmitting datagrams. This parameter is only valid for DVP.

$A$ : cost per VC opening.

$B$ : buffering cost per unit time per IP datagram present in the system.

$R$ : cost per unit time for running the VC.

\section{ANALYSIS OF DVP}

In this section, we evaluate the cost function of the DVP and evaluate the delay time $T$ to minimize the cost of the DVP. In the DVP, the signalling process required to open a VC starts as soon as a packet arrives at the IP-ATM interface. However, when all the packets arriving at the IP-ATM interface have been transmitted, instead of the VC being closed immediately, it is left open for a time $T$ in anticipation of more packets to arrive at the IP-ATM gateway. If no packets arrive within time $T$, the $\mathrm{VC}$ is closed down, i.e. the server vacation is delayed by time $T$; hence the name delayed vacation policy.

\subsection{Cost function of DVP}

The cost of operating VCs in the DVP policy depends on the running cost of the VC and the VC opening cost. The VC running cost consists of a component incurred during transmission of packets and a cost due to idling of VCs. The later component of the cost can be minimised by chosing a low value of $T$, which unfortunately increases the VC opening cost due to more frequent VC setups. The challenge is to determine the optimum value of the timer $T$ which will minimise the operating cost of the DVP. In this section, we derive an expression for the optimum timer value $T$.

The cost function $C_{d}(T)$ in the DVP can be expressed $a s^{6}$ :

$$
C_{d}(T)=A \gamma+B L+R(\rho+\pi), \quad T \geq 0 .
$$

where the first term accounts for $\mathrm{VC}$ opening cost, the second term for buffering cost, and the third term for VC running cost.

Using the expressions derived for $\gamma$ and $\pi$ in, ${ }^{3}$ and for $L$ in,${ }^{4}$ we obtain for an $M / G / 1$ queue:

$$
\begin{aligned}
C_{d}(T)= & \frac{A \lambda e^{-\lambda T}(1-\rho)}{1+\lambda S e^{-\lambda T}}+B\left[\rho+\frac{\lambda^{2} b_{2}}{2(1-\rho)}+\frac{\lambda S e^{-\lambda T}(2+\lambda S)}{2\left(1+\lambda S e^{-\lambda T}\right)}\right]+ \\
& R\left[\rho+\frac{(1-\rho)\left(1-e^{-\lambda T}\right)}{1+\lambda S e^{-\lambda T}}\right], T \geq 0
\end{aligned}
$$

where $b_{2}$ is the second moment of service time distribution. $C_{d}(T)$ for an $\mathrm{M} / \mathrm{M} / 1$ queue can be obtained by replacing $b_{2}$ with $\frac{1}{\mu^{2}}$ in Eqn. 2 . 


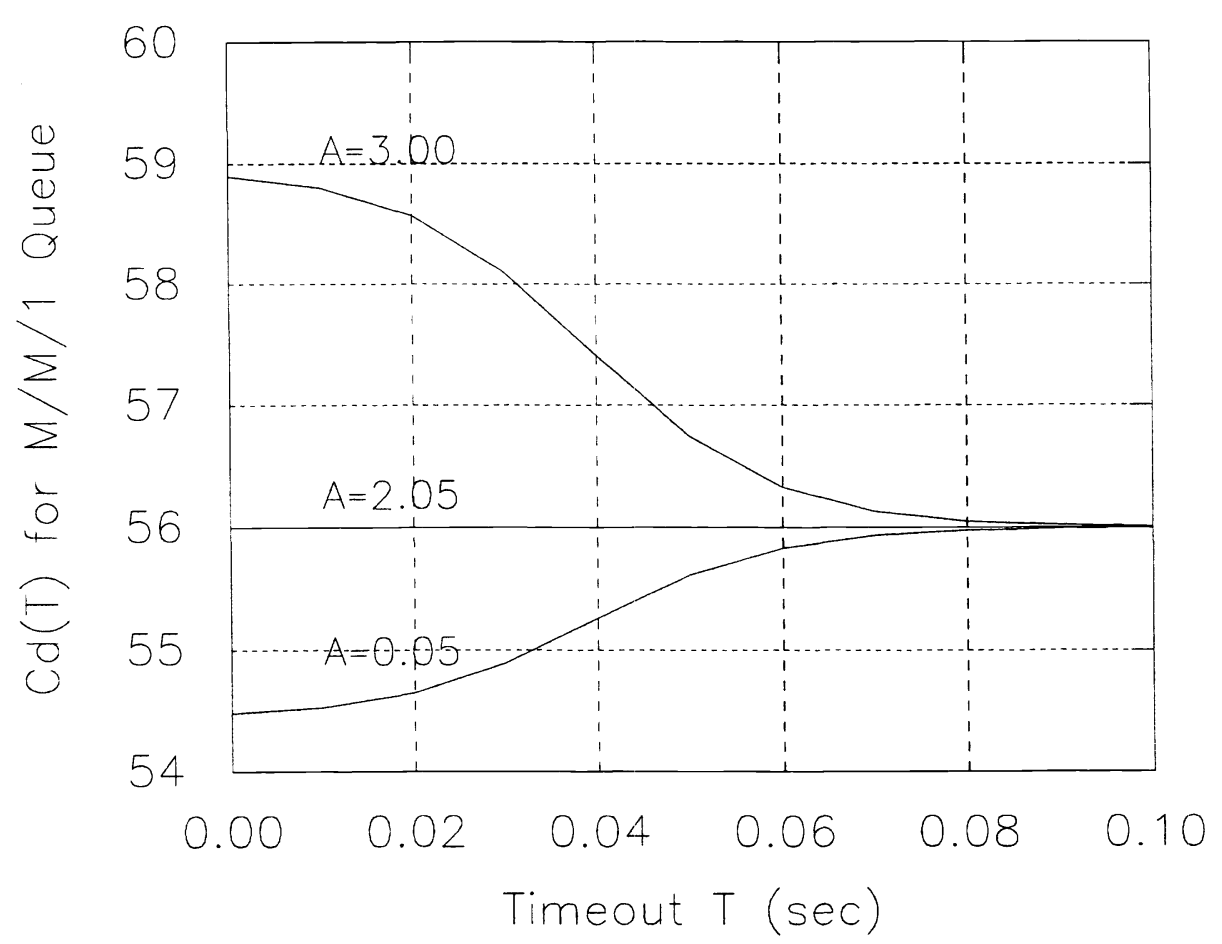

Figure 2. Illustration of $C_{d}(T)$ for the three conditions of $d C_{d}(T) / d T$ for $\lambda=100, \rho=0.5, S=0.5, B=1$ and $R=55$.

\subsection{Optimal Control for DVP}

To determine the optimal value of $T$ in order to minimise the cost function for DVP, the first derivative of $C_{d}(T)$ is examined to investigate the existence of any minima for $C_{d}(T)$. After simplification, the first derivative of $C_{d}(T)$ is given by,

$$
\frac{d C_{d}(T)}{d T}=\left[\frac{\lambda e^{-\lambda T}}{2\left(1+\lambda S e^{-\lambda T}\right)^{2}}\right][-2 A \lambda(1-\rho)-B \lambda S(2+\lambda S)+2 R(1-\rho)(1+\lambda S)] .
$$

Eqn. (3) has two factors in the RHS. The first factor is always positive and hence the sign of $d C_{d}(T) / d T$ depends only on the sign of the second factor. Considering the second factor only, we have the following three cases:

$$
\frac{d C_{d}(T)}{d T}= \begin{cases}+v e, & A x+B y+R z>0 \\ 0, & A x+B y+R z=0 \\ -v e, & A x+B y+R z<0\end{cases}
$$

where $x=-2 \lambda(1-\rho), y=-\lambda S(2+\lambda S)$, and $z=2(1-\rho)(1+\lambda S)$. For positive $d C_{d}(T) / d T, C_{d}(T)$ is an increasing function which asymptotically approaches $R+B\left(\rho+\frac{\lambda^{2} b_{2}}{2(1-\rho)}\right)$ as $T$ approaches infinity. For negative $d C_{d}(T) / d T$, $C_{d}(T)$ is a decreasing function which asymptotically approaches $R+B\left(\rho+\frac{\lambda^{2} b_{2}}{2(1-\rho)}\right)$ as $T$ approaches infinity, and for zero $d C_{d}(T) / d T, C_{d}(T)$ is a constant equals to $R+B\left(\rho+\frac{\lambda^{2} b_{2}}{2(1-\rho)}\right)$. Figure 2 shows $C_{d}(T)$ versus $T$ for three values of $A$ to illustrate the three conditions described in Eqn. (4). We note that $C_{d}(T)$ is either an increasing or a decreasing function without any finite positive $T$ for which $C_{d}(T)$ has a minima. $C_{d}(T)$ is minimised at:

$$
\hat{T}= \begin{cases}0, & A x+B y+R z \geq 0 \\ \infty, & A x+B y+R z<0\end{cases}
$$

Therefore, the optimal operating policy for DVP is either to close the VC as soon as the last datagram from the queue is transmitted $(T=0)$, or always leave the VC open $(T=\infty)$ corresponding to a PVC. 


\section{COST OF PVC}

In this section, we determine the cost $C_{p}$ of operating the VC as as PVC. We then use the cost to compare against the cost of operating the VC under the COP in Section 6.2. For a PVC, $\gamma=0, \rho+\pi=1$ and $L=\rho /(1-\rho)$ in Eqn 1, and hence the operating cost of a PVC is simply $R+B L$. For an M/M/1 queue the operating cost of a PVC $\left(C_{p}\right)$ is obtained by setting $\gamma=0, \rho+\pi=1$ and the value of $L$ (from $\left.{ }^{4}\right)$ in Eqn. (1).

$$
C_{p}=R+\frac{B \rho}{1-\rho}
$$

\section{ANALYSIS OF COP}

In the Control Operating Policy (COP), the server goes to vacation whenever it is idle. As soon as $K$ packets have accumulated in the queue, the server starts the VC connection setup process and then transmits the packets. In this section, we derive the cost function for the COP which will then be compared with the cost of operating the VC under the DVC and PVC in Section 6.

\subsection{Cost Function of COP}

Since the VC never remains idle under the COP, we have $\pi=0$ and the cost function $C_{c}(K)$ of the COP as a function of the queue threshold $K$ is given by:

$$
C_{c}(K)=A \gamma+B L+R \rho, \quad K=1,2, \ldots
$$

Under the COP, the VC opening rate is equivalent to the number of times the queueing system becoms idle. Therefore, we can express the VC opening rate as ${ }^{11}$ :

$$
\gamma=\frac{\lambda(1-\rho)}{K+\lambda S}
$$

For an $\mathrm{M} / \mathrm{M} / 1$ queue under COP with constant 'startup' time (VC opening delay), the average queue length $L$ can be obtained as, ${ }^{8}$

$$
L=\frac{1}{2(K+\lambda S)}\left[K(K-1)+2 K \lambda S+\lambda^{2} S^{2}\right]+\frac{\rho^{2}}{1-\rho} .
$$

Substituting the expressions for $\gamma$ and $L$ from Eqns. (8) and (9) into Eqn. (7) and after simplication, we obtain the cost function for the COP as:

$$
C_{c}(K)=\frac{A \lambda(1-\rho)}{K+\lambda S}+\frac{B}{2(K+\lambda S)}\left[K(K-1)+2 K \lambda S+\lambda^{2} S^{2}\right]+\rho\left(R+\frac{B \rho}{1-\rho}\right), \quad K=1,2, \ldots
$$

\subsection{Optimal Control for COP}

In this section, we study the behaviour of function $C_{c}(K)$ (as given by Eqn. (10)) to investigate the existence of $\hat{K}$, a positive integer which minimises (optimises) $C_{c}(K)$. Although $C_{c}(K)$ is a discrete function valid only at $K=1,2, \ldots$, it helps to obtain $\hat{K}$ by treating $C_{c}(K)$ as a continuous function. Differentiating $C_{c}(K)$ with respect to $K$ and setting $d C_{c}(K) / d K=0$, we obtain

$$
K_{1}=-\lambda S-\sqrt{\lambda S+2 \frac{A}{B} \lambda(1-\rho)}
$$

and

$$
K_{2}=-\lambda S+\sqrt{\lambda S+2 \frac{A}{B} \lambda(1-\rho)}
$$

where $K_{1}$ and $K_{2}$ are two roots of $d C_{c}(K) / d K=0 . K_{1}$ is always negative and is not of our interest, as $C_{c}(K)$ is only valid for $K \geq 1$. Therefore, we only consider $K_{2}$. We observe the following two cases:

1. $\frac{A}{B} \leq \frac{\lambda S(\lambda S-1)}{2 \lambda(1-\rho)}$. In this case, $K_{2} \leq 1$ meaning $C_{c}(K)$ is an increasing function for $K \geq 1$; for this case $\hat{K}=1$.

2. $\frac{A}{B}>\frac{\lambda S(\lambda S-1)}{2 \lambda(1-\rho)}$. In this case, $K_{2}>1$ and it can be shown (see Appendix) that $C_{c}(K)$ is a convex function for $K \geq 1$ with the minima occuring at $K_{2}$. For this case, $\hat{K}=\left\lfloor K_{2}\right\rfloor$ or $\left\lceil K_{2}\right\rceil$. 


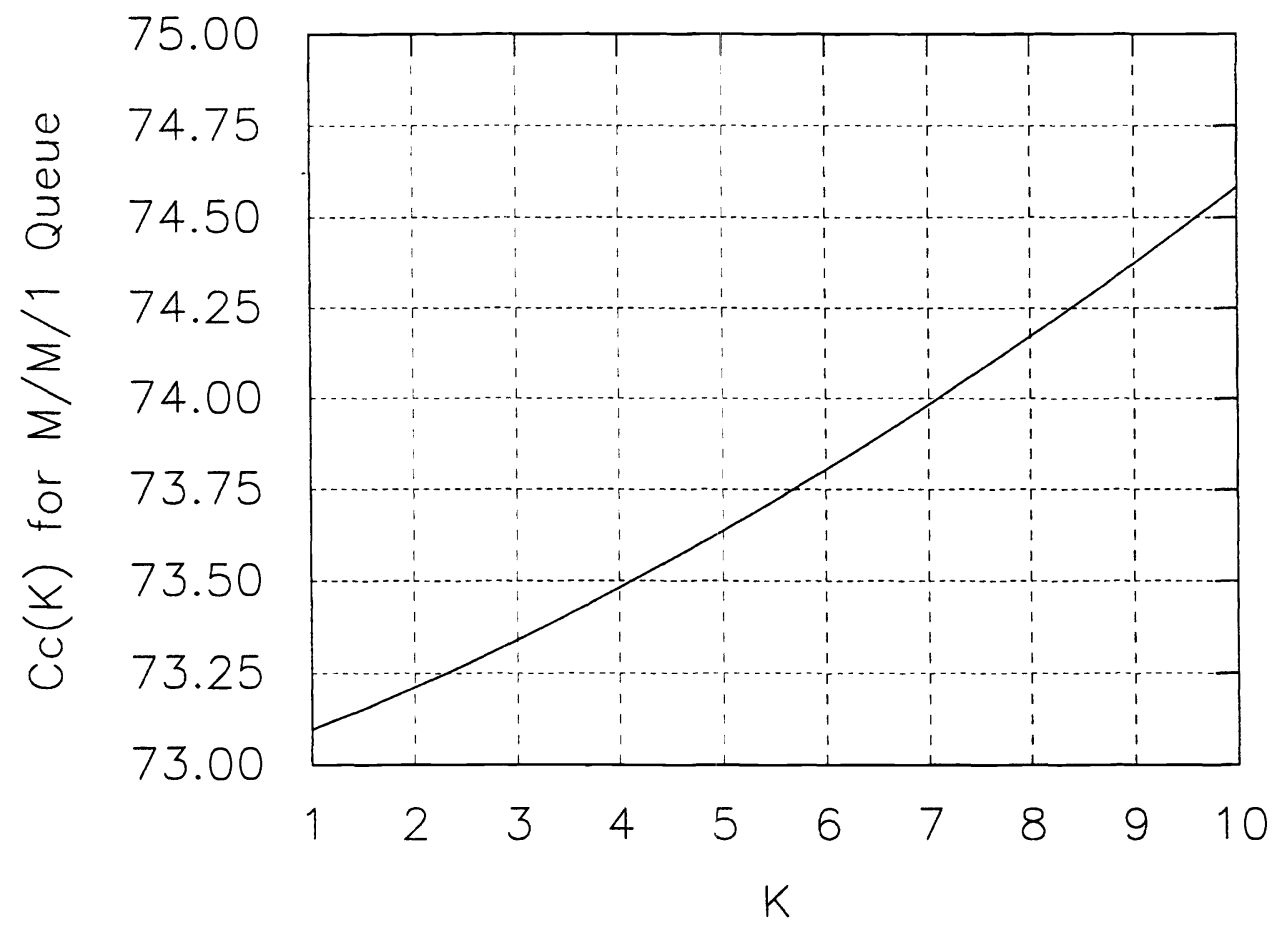

Figure 3. $C_{c}(K)$ for $K>1$ corresponding to $A=20, \lambda=100, \rho=0.5, S=0.5, B=1, R=55$.

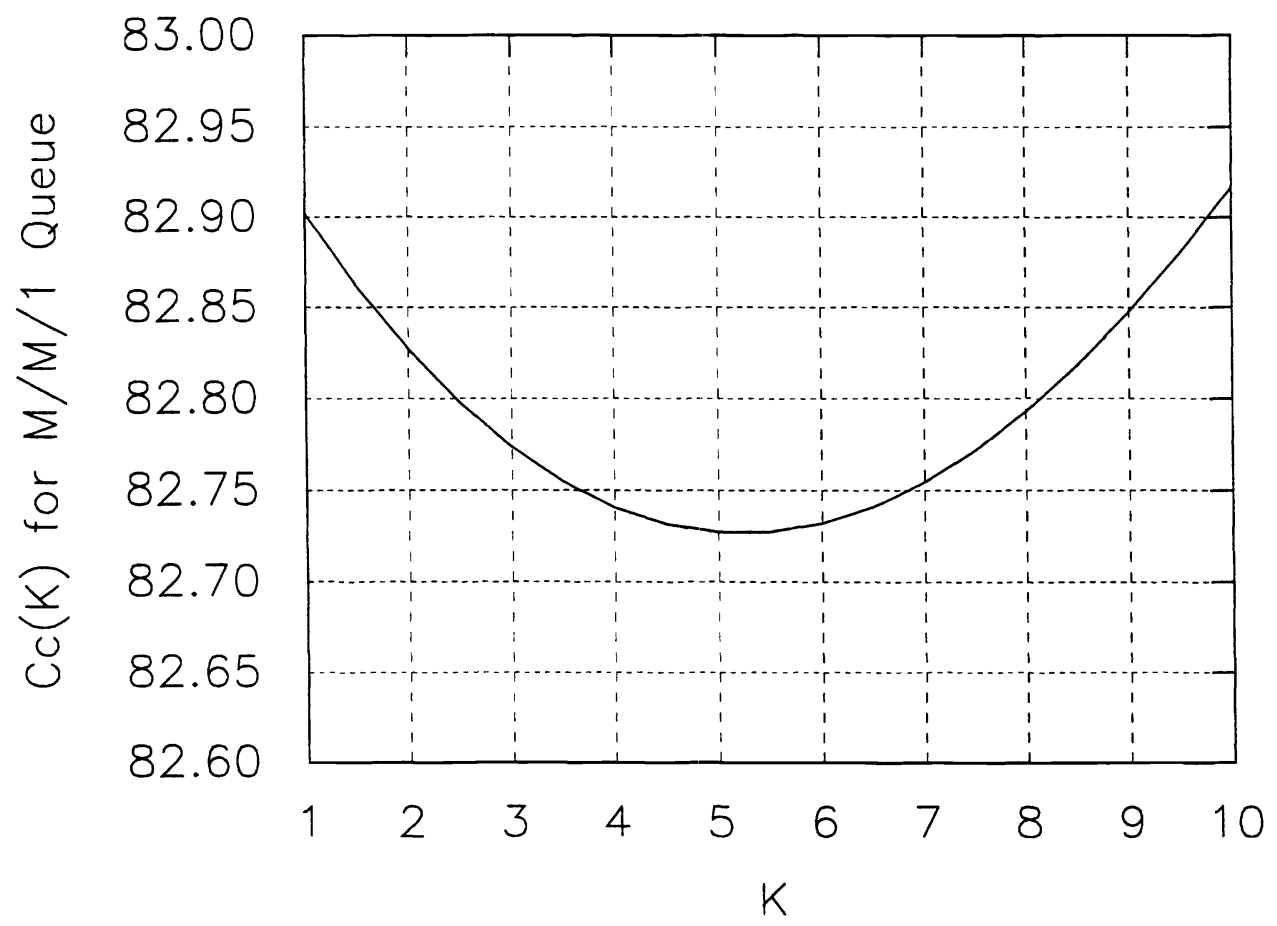

Figure 4. $C_{c}(K)$ for $K_{2} \leq 1$ corresponding to $A=30, \lambda=100, \rho=0.5, S=0.5, B=1, R=55$. 
To verify our observations above regarding the nature of $C_{c}(K)$ described above, Figures 3 and 4 show $C_{c}(K)$ for the two conditions mentioned above.

For certain values of the cost coefficients $(A, B$ and $R)$, it may turn out that $C_{p} \leq C_{c}(\hat{K})$. For those values of the coeffecients, the optimum operating policy will be to manage the VC as a PVC.

It is interesting to note that, for $S=0$, the right hand side of Eqn. (12) reduces to the expression obtained in ${ }^{12}$ for zero 'startup time' (equivalent to zero VC opening delay) under the same cost structure as used in this paper. This also verifies the correctness of our equations.

\section{COMPARISON OF THE SCHEMES}

In the previous sections, we have modeled the COP as applied to the the connection management of a switched virtual circuit for IP-ATM internetworking. In this section, we comapare the cost of a VC operating under the COP against the costs of operating a VC using DVP and PVC.

\subsection{Comparison beween COP and DVP}

As mentioned in Section 3, the optimal operating policy under DVP is either

- operating at $T=\infty$ (PVC), or

- operating at $T=0$ which is equivalent to $\mathrm{COP}$ at $K=1$.

Therefore, the minimum cost incurred under COP will be lower than the minimum cost incurred under DVP if there exists a $\hat{K}>1$.

In order to have $\hat{K}>1$, we must have $K_{2}>1$. However, $K_{2}>1$ does not guarantee that $\hat{K}>1$, as $\hat{K}$ may be obtained as $\left\lfloor K_{2}\right\rfloor$. To guarantee a $\hat{K}>1$, it is required to have $K_{2} \geq 2$. In order to have $K_{2} \geq 2$, we need to have (from Eqn. (12))

$$
\frac{A}{B} \geq \frac{4+\lambda S(\lambda S+3)}{2 \lambda(1-\rho)}
$$

The above equation, therefore, sets the condition to achieve a guaranteed lower operating cost of the COP over the DVP.

\subsection{Comparison beween COP and PVC}

In this subsection we evaluate the conditions under which the operating cost of COP will be less than the PVC, i.e. the conditions for which $C_{C}(\hat{K})<C_{p}$. Eqn. (10) can be written as:

$$
C_{c}(\hat{K})=Y(\hat{K})+\rho\left(R+\frac{B \rho}{1-\rho}\right), \quad \hat{K}=1,2, \ldots
$$

Substituting Eqn (6) in the above equation gives

$$
C_{c}(\hat{K})=Y(\hat{K})+\rho C_{p}, \quad \hat{K}=1,2, \ldots
$$

From Eqn. (15), in order to have $C_{C}(\hat{K})<C_{p}$, we need to have

$$
R>\frac{Y(\hat{K})-B \rho}{1-\rho} .
$$

Eqn (16) therefore sets the condition to achieve a lower operating cost of the COP over PVC.

\subsection{Comparison of DVP, COP and PVC}

Having inidividually derived the conditions (Eqns 13 and 16) under which COP will achieve a lower cost than DVP and PVC, we now combine both of the conditions to achieve a lower operating cost for the COP as comapared to the other two policies. Therefore, an $\mathrm{M} / \mathrm{M} / 1$ queue under COP achieves lower cost than both $P V C$ and DVP when the following condition is met:

$$
\frac{A}{B} \geq \frac{4+\lambda S(\lambda S+3)}{2 \lambda(1-\rho)} \text { and } R>\frac{Y(\hat{K})-B \rho}{1-\rho}
$$

where $\hat{K}=\left\lfloor K_{2}\right\rfloor$ or $\left\lceil K_{2}\right\rceil$ as discussed in Section 5.2 


\section{CONCLUSION}

We have conisdered three different policies for managing the virtual circuit at an IP-ATM interface and derived models for the cost functions of the policies. We have also derived conditions under which each of the policies is individually optimal. Subsequently, given a set of traffic (such as arrival pattern of packets at the interface) and network parameters (such as the VC opening cost and delay, VC running cost, etc.), we have combined the individual conditions to determine the most cost effective policy to use for the set of parameters. We have also derived the condition for which the Control Operating Policy will be cost effective than the Delayed Vacation Policy or a Permanent Virtual Circuit. Our study will enable telecommunication carriers to optimise their cost of transporting IP traffic over ATM networks.

\section{Appendix : Minima for $C_{c}(K)$}

From Eqns. (11) and (12), we can say that the sign of $d C_{c}(K) / d K$ is determined by the sign of $\left(K-K_{1}\right)\left(K-K_{2}\right)$. For $K_{2}>1$ we observe the following.

For $K>K_{2}$, both $\left(K-K_{1}\right)$ and $\left(K-K_{2}\right)$ are positive and hence the sign of $d C_{c}(K) / d K$ is positive meaning $C_{c}(K)$ is an increasing function. However, for $1 \leq K<K_{2},\left(K-K_{1}\right)$ is positive, but $\left(K-K_{2}\right)$ is negative and hence $d C_{c}(K) / d K$ is negative meaning $C_{c}(K)$ is a decreasing function. Therefore, $C_{c}(K)$ is a convex function with the minima occuring at $K_{2}$.

\section{REFERENCES}

1. M. Perez, F. Liaw, A. Mankin, E. Hoffman, D. Grossman, and A. Malis, RFC 1755: ATM Signalling Support for IP over ATM, February 1995.

2. S. Keshav, C. Lund, S. Phillip, N. Reingold, and H. Saran, "An Empirical Evaluation of Virtual Circuit Holding Policies in IP-Over-ATM Networks," IEEE JSAC 13, pp. 1371-1382, October 1995.

3. M. Hassan and M. Atiquzzaman, "A Delayed Vacation Model of an M/G/1 Queue with Setup Time and Its Application to SVCC-Based ATM Networks," IEICE Transactions on Communications E80-B, pp. 317-323, February 1997.

4. H. Kitazume and Y. Takahashi, "Waiting-time distribuion in a single-server queue with vacation/set-up/closedown times for IP over ATM networks," in PMCCN'97 Workshop, pp. 58-65, (Tsukuba, Japan), Nov 21, 1997.

5. Y. Sakai, Y. Takahashi, Y. Takahashi, and T. Hasegawa, "A composite queue with vacation/set-up/close-down times for SVCC in IP over ATM networks," Journal of the Operations Research Society of Japan 41, pp. 68-80, March 1998.

6. M. Hassan, "Economic Behaviour of an M/G/1 Queue under Delayed Vacation Policy," Tech. Rep. 2/98, Monash University, Churchill, VIC 3842, Australia, March 1998.

7. D. P. Heyman., "Optimal Operating Policies for M/G/1 Queuing Systems," Operations Research 16, pp. 362$382,1968$.

8. B. et al., "Poisson Input Queueing System with Startup Time and Under Control Operating Policy," Comp. Opns. Res. 14, pp. 33-40, 1987.

9. R. Hwang and J. Kurose, "On-Call Processing Delay in High Speed Networks," IEEE/ACM Transactions on Networking 3, pp. 628-639, December 1995.

10. D. Niehaus, A. Battou, A. McFarland, B. Decina, V. Sirkay, and B. Edwards, "Performance Benchmarking of Signalling in ATM Networks," IEEE Communications Magazine, pp. 134-143, August 1997.

11. J. Medhi, Stochastic Models in Queueing Theory. Academic Press, 1991.

12. H. C. Tijms, Stochastic Modelling and Analysis: A Computational Aspect, Wiley, 1986. 